\title{
Identificação dos Parâmetros e Simulação de um Quadrotor Construído por meio de Prototipação Rápida
}

\author{
Eduardo Sacogne Fraccaroli ${ }^{1}$ \\ Instituto de Ciências Matemáticas e de Computação, USP, São Carlos, SP \\ Roberto Santos Inoue ${ }^{2}$ \\ Departamento de Engenharia Elétrica, UFSCAR, São Carlos, SP \\ Isabella Cristina Souza Faria ${ }^{3}$ \\ Departamento de Engenharia Mecânica, UFSCAR, São Carlos, SP \\ Roseli Aparecida Francelin Romero ${ }^{4}$ \\ Instituto de Ciências Matemáticas e de Computação, USP, São Carlos, SP
}

\begin{abstract}
Resumo. O quadrotor LARVANTAR é um projeto do Instituto de Ciências Matemáticas e de Computação da USP de São Calos para o desenvolvimento de uma aeronave através de prototipagem rápida que realize o rastreamento de veículos terrestres, capture imagens aéreas e faça a coleta de dados de uma plantação de laranjas. Atualmente essa aeronave é controlada por um piloto automático Naza V2 desenvolvido pela empresa DJI. Mas pretendese substituir esse controlador por um piloto automático próprio. Para isso, nesse trabalho foram identificados os parâmetros do modelo dinâmico do quadrotor com auxílio de um software CAD. Um controlador PD foi projetado para o problema de acompanhamento de trajetória da aeronave. E resultados simulados do voo do veículo foram obtidos para demonstrar o desempenho do controle que será embarcado no quadrotor.
\end{abstract}

Palavras-chave. Quadrotor, VANT, controle PD, controle de quadrotor, desenvolvimento de quadrotor.

\section{Introdução}

Veículos aéreos não tripulados (VANT), vem sendo utilizados por diversos setores da economia, como as áreas agrícola, industrial, comercial e predial. Isto ocorreu principalmente devido a crescente disponibilidade de recursos computacionais de alto desempenho, avanços em tecnologias de transmissão de dados e de posicionamento global. O que permitiu o desenvolvimento de aeronaves mais confiáveis e versáteis e a diminuição dos custos de construção desses veículos, [11] e [13].

\footnotetext{
1eduardo.fraccaroli@gmail.com

2rsinoue@ufscar.br

${ }^{3}$ isamoreno2009@gmail.com

${ }^{4}$ rafrance@icmc.usp.br
} 
Um dos principais tipos de VANTs são os quadrotores. Esse tipo de aeronave vem sendo utilizado para navegação autônoma visual em ambientes não estruturados, [1], vigilância autônoma, [6], assistente em esporte, [8], enxames de robôs, [4] e [12], em impressão 3D aérea, [9], etc. Contudo a construção desse tipo de veículo não é tarefa fácil, pois envolve o projeto e desenvolvimento da estrutura mecânica, eletrônica, sistema embarcado e sistema de controle. Por exemplo, em [4] foi desenvolvido um quadrotor de baixo custo para realização de experimentos de enxame de robôs em ambiente interno, nesse trabalho foi utilizado um piloto automático de código aberto o ArdupilotMega 2.0 e o sistema de captura de movimento da Vicon para realizar a realimentação do sistema de controle. Já, outros trabalhos focam no desenvolvimento de controladores PD para o problema de acompanhamento de trajetória desse tipo de veículo, [10], [5], [14] e [2].

Assim, nesse artigo é apresentado o desenvolvimento de um quadrotor constituído por hastes de alumínio e peças prototipadas por uma impressora $3 \mathrm{D}$ de baixo custo. A identificação dos parâmetros do modelo dinâmico dessa aeronave foram obtidos através da utilização de um software CAD, [7]. O projeto de controlador PD para o problema de acompanhamento de trajetória do quadrotor foi desenvolvido e a simulação do voo do veículo foi realizada para demonstrar o desempenho do controle utilizado.

\section{Quadrotor LARVANTAR}

Este quadrotor foi projetado e desenvolvido como plataforma padrão para ser utilizado em diversas aplicações, dentre elas: imageamento aéreo, coleta de dados de uma plantação de laranjas e rastreamento de veículos. Foi utilizado uma impressora 3D, para prototipar em plástico ABS suas peças estruturais.

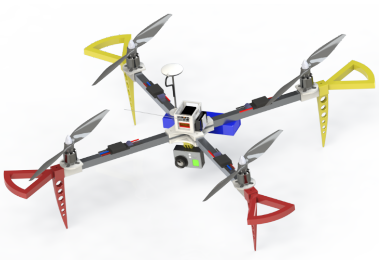

(a)

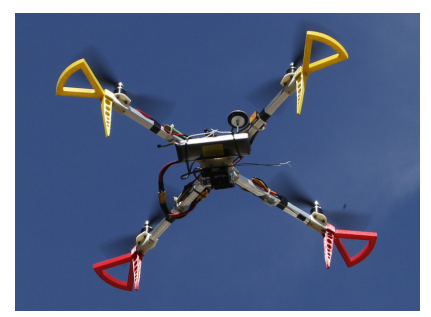

(b)

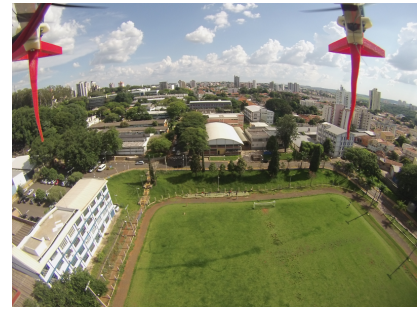

(c)

Figura 1: Modelo 3D do quadrotor 1(a), quadrotor em voo 1(b) e imagem aérea do Campus 1 da USP de São Carlos 1(c).

O quadrotor, Figura 1(b), possui um piloto automático que o controla por meio da fusão dos dados dos sensores (IMU e GPS). Para realizar a captura de imagens aérea, Figura 1(c), foi acoplado uma câmera GoPro Hero 3.

Atualmente esse quadrotor possui dois modos de voo: voo autônomo ou voo rádio controlado. No voo autônomo utilizamos um tablet para controle, onde via bluetooth envia comandos à uma estação situada em terra, que por sua vez envia os comandos para o quadrotor. No voo rádio controlado utilizamos um controle com 6 canais para controlar 
os movimentos do quadrotor.

A eletrônica é composta de quatro módulos responsáveis pelo controle eletrônico de velocidade, cada um com a corrente máxima de operação de 30A; quatro motores brusheless de $750 \mathrm{kv}$ de potência; duas hélices que giram no sentido horário e duas hélices que giram no sentido anti-horário; uma bateria LIPO $3 \mathrm{~S}$ com 4200mAh e 60C; um módulo Naza V2 $(\mathrm{MC}+\mathrm{PMU}+\mathrm{LED})$ e um GPS.

O chassi do quadrotor possui quatro tubos quadrados em alumínio (hastes) e o restante são peças prototipadas. O projeto das peças prototipadas foi desenvolvido para atender as necessidades das aplicações onde será inserido o quadrotor. Dentre as peças impressas o corpo é a peça principal onde as quatro hastes são fixadas. O suporte do motor foi desenhado para ser vestido na haste, propiciando assim nenhuma folga e melhor fixação. $\mathrm{O}$ protetor de hélice foi desenhado para ser inserido sem folga dentro da haste e aparafusado. O trem de pouso foi desenhado para deixar um vão livre entre o chão e o corpo do quadrotor. A caixa de proteção da eletrônica foi desenhada para encapsular o módulo Naza V2 deixando suas portas livres para conexão dos cabos de alimentação e cabos de dados. O suporte da GoPro foi adaptado para se aquedar ao corpo do quadrotor. Optou-se por utilizar peças prototipadas por três motivos: custo de fabricação reduzido; facilidade de reposição das peças; e redução do tempo do ciclo de desenvolvimento do projeto.

\section{Obtenção dos parâmetros}

A obtenção dos parâmetros do modelo dinâmico do quadrotor LAVANTAR consistiu na representação da aeronave em software CAD, Figura 1(a). Cada peça do veículo foi desenhada separadamente e com especificação do material. Em seguida, foi feita a montagem e obteve-se as propriedades do conjunto, como a massa $m$, os momentos de inércia com relação aos três eixos $I_{x x}, I_{y y}$ e $I_{z z}$ e a distância dos rotores até o centro de massa do veículo $l$. Os dados obtidos do quadrotor são mostrados na Figura 2(a) e os valores de ganho do controlador PD de acordo com [10], são apresentados na Figura $2(b)$.

\begin{tabular}{clr}
\hline Parâmetro & Valor & Unidade \\
\hline $\mathrm{g}$ & 9.81 & $\mathrm{~m} / \mathrm{s}^{2}$ \\
$\mathrm{~m}$ & 1.221 & $\mathrm{Kg}$ \\
$I_{x x}$ & $3.16718^{-3}$ & $\mathrm{Kg} m^{2}$ \\
$I_{y y}$ & $6.16466^{-3}$ & $\mathrm{Kg} m^{2}$ \\
$I_{z z}$ & $3.23107^{-3}$ & $\mathrm{Kg} m^{2}$ \\
\hline
\end{tabular}

(a)

\begin{tabular}{cl}
\hline Parâmetro & Valor \\
\hline$K_{z d}$ & 2.5 \\
$K_{\phi d}$ & 1.75 \\
$K_{\theta d}$ & 1.75 \\
$K_{\psi d}$ & 1.75 \\
$K_{z p}$ & 1.5 \\
$K_{\phi p}$ & 6 \\
$K_{\theta p}$ & 6 \\
$K_{\psi p}$ & 6 \\
\hline
\end{tabular}

(b)

Figura 2: Parâmetro identificados através do modelo 3D 1(a) e parâmetros de ganho do controlador PD 2(b). 


\section{Modelagem e controle do quadrotor}

O quadrotor é considerado como um corpo rígido e então, por meio das equações de Newton-Euler, pode-se descrever sua dinâmica, [10]. A equação para as acelerações angulares é dada por:

$$
\ddot{n}=\left[\begin{array}{c}
\ddot{\phi} \\
\ddot{\theta} \\
\ddot{\psi}
\end{array}\right]=J^{-1}\left(\tau_{B}-C \dot{n}\right),
$$

sendo $\ddot{\phi}_{d}$ e $\ddot{\theta}_{d}$ e $\ddot{\psi}_{d}$ as acelerações angulares desejadas de rolagem, arfagem e guinada, $J$ a matriz Jacobina, $\tau_{B}=\left[\begin{array}{lll}\tau_{\phi} & \tau_{\theta} \tau_{\psi}\end{array}\right]^{T}$ o vetor de torque total, $\tau_{\phi}$ o torque gerado pela rotação em torno do eixo $x, \tau_{\theta}$ o torque gerado pela rotação em torno do eixo $y, \tau_{\psi}$ o torque gerado pela rotação em torno do eixo $z$ e $C$ a matriz de Coriolis. A equação para as acelerações lineares é dada por:

$$
\ddot{s}=\left[\begin{array}{c}
\ddot{x} \\
\ddot{y} \\
\ddot{z}
\end{array}\right]-g\left[\begin{array}{l}
0 \\
0 \\
1
\end{array}\right]+\frac{T}{m}\left[\begin{array}{c}
\cos \psi \sin \theta \cos \phi+\sin \psi \sin \phi \\
\sin \psi \sin \theta \cos \phi-\cos \psi \sin \phi \\
\cos \theta \cos \phi
\end{array}\right]-\frac{1}{m}\left[\begin{array}{ccc}
A_{x} & 0 & 0 \\
0 & A_{y} & 0 \\
0 & 0 & A_{z}
\end{array}\right]\left[\begin{array}{c}
\dot{x} \\
\dot{y} \\
\dot{z}
\end{array}\right],
$$

sendo $\ddot{x}, \ddot{y}$, e $\ddot{z}$ as acelerações lineares nos eixos $x, y$, e $z$, respectivamente, $g$ a constante gravitacional, $T$ o impulso total gerado pelas forças combinadas dos rotores, e $A_{x}, A_{y}$ e $A_{z}$ os coeficientes da força do vento nas correspondentes direções do referencial inercial,.

As equações geradas pelo controlador PD são dadas por:

$$
\begin{aligned}
T & =\left(g+\left(K_{z d}\right)\left(\dot{z}_{d}-\dot{z}\right)+\left(K_{z p}\right)\left(z_{d}-z\right)\right) \frac{m}{(\cos \phi \cos \theta)}, \\
\tau_{\phi} & =\left(K_{\phi d}\left(\dot{\phi}_{d}-\dot{\phi}\right)+K_{\phi p}\left(\phi_{d}-\phi\right)\right) I_{x x}, \\
\tau_{\theta} & =\left(K_{\theta d}\left(\dot{\theta}_{d}-\dot{\theta}\right)+K_{\theta p}\left(\theta_{d}-\theta\right)\right) I_{y y} \\
\tau_{\psi} & =\left(K_{\psi d}\left(\dot{\psi}_{d}-\dot{\psi}\right)+K_{\psi p}\left(\psi_{d}-\psi\right)\right) I_{z z},
\end{aligned}
$$

sendo $K_{z d}, K_{\phi d}, K_{\theta d}$ e $K_{\psi d}$ os ganhos derivativos, $K_{z p}, K_{\phi p}, K_{\theta p}$ e $K_{\psi p}$ os ganhos proporcionais; $\dot{z}_{d}$ a velocidade desejada no eixo $z, z_{d}$ a posição desejada no eixo $z ; \phi_{d}, \theta_{d}$ e $\psi_{d}$ os ângulos desejados de rolagem, arfagem e guinada, e $\dot{\phi}_{d}$ e $\dot{\theta}_{d}$ e $\dot{\psi}_{d}$ as velocidades angulares desejadas de rolagem, arfagem e guinada.

A trajetória desejada $x_{d}, y_{d}$ e $z_{d}$ é obtida através de um polinômio de quinto grau, [3]. E os ângulos desejados por:

$$
\begin{aligned}
& \phi_{d}=a s\left(\frac{d_{x} \sin (\psi)-d_{y} \cos (\psi)}{d_{x}^{2}+d_{y}^{2}+\left(d_{z}+g\right)^{2}}\right), \\
& \theta_{d}=a t\left(\frac{d_{x} \cos (\psi)+d_{y} \sin (\psi)}{d_{z}+g}\right),
\end{aligned}
$$

sendo

$$
\begin{aligned}
d_{x} & =K_{x p}\left(x_{d}-x\right)+K_{x d}\left(\dot{x}_{d}-\dot{x}\right)+K_{x d d}\left(\ddot{x}_{d}-\ddot{x}\right), \\
d_{y} & =K_{y p}\left(y_{d}-y\right)+K_{y d}\left(\dot{y}_{d}-\dot{y}\right)+K_{y d d}\left(\ddot{y}_{d}-\ddot{y}\right), \\
d_{z} & =K_{z p}\left(z_{d}-z\right)+K_{z d}\left(\dot{z}_{d}-\dot{z}\right)+K_{z d d}\left(\ddot{z}_{d}-\ddot{z}\right) .
\end{aligned}
$$




\section{Simulação e Análise dos resultados}

A simulação do controle do quadrotor foi implementada em MATLAB ${ }^{\circledR}$, conforme ilustrado pelo diagrama da Figura 3. Primeiramente foi gerada uma trajetória desejada para o quadrotor através de um polinômio do quinto grau, que irá traçar uma reta entre a posição original do quadrotor e a posição final desejada do quadrotor.

Essa trajetória é então comparada com a posição do quadrotor ao longo do tempo. Um erro entre a trajetória desejada e a posição atual do quadrotor é calculado. Esse erro é então utilizado para o cálculo do controlador PD, que fornece o impulso total e o torque total para o modelo do quadrotor. E através de uma dupla integração, obtém-se o valor de posição e atitude do quadrotor.

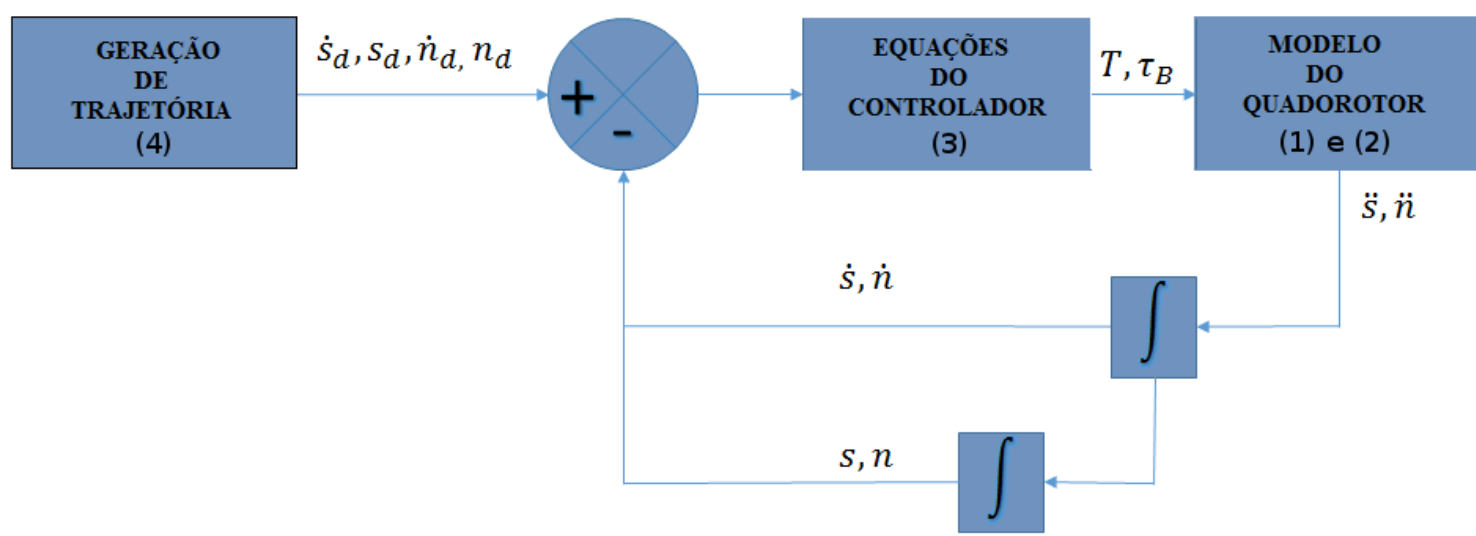

Figura 3: Diagrama de blocos do sistema de controle do quadrotor LARVANTAR.

Para simulação foram utilizados os valores dos parâmetros baseados em [10] e nos momentos de inercia do quadrotor real (Figura 2(a)).

As figuras 4(a), 4(b) e 4(c) mostram as posições lineares e as figuras 5(a), 5(b) e 5(c) mostram as posições angulares resultantes da simulação.

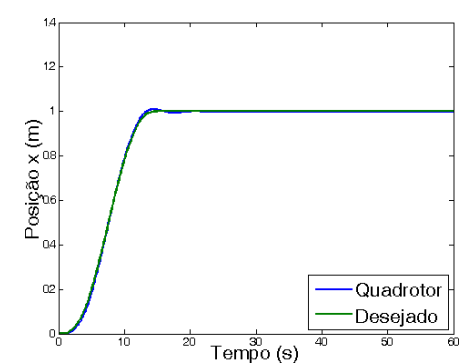

(a)

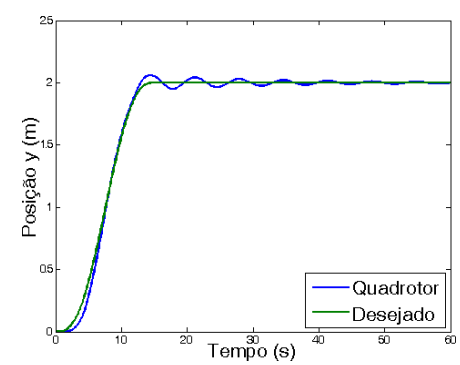

(b)

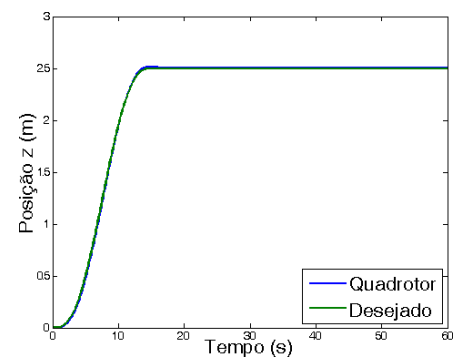

(c)

Figura 4: Posições lineares de de rolagem 4(a), arfagem 4(b) e guinada 4(c).

A simulação mostrou que a modelagem do quadrotor foi satisfatória. Além disso, o controlador $\mathrm{PD}$ funcionou bem, porém, houve uma variação dos valores de $x$ e $y$ durante o 


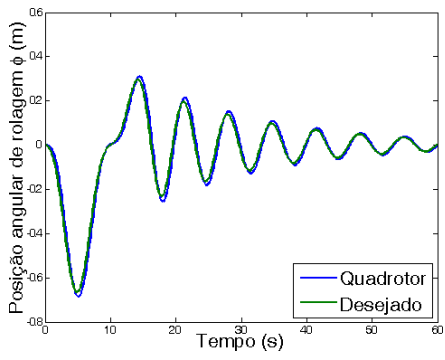

(a)

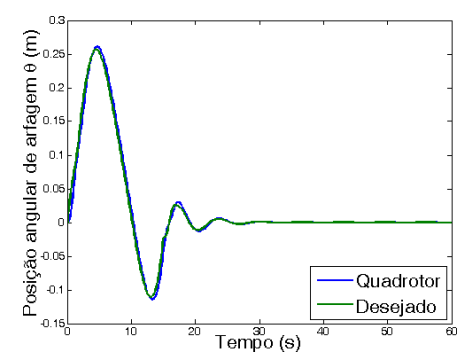

(b)

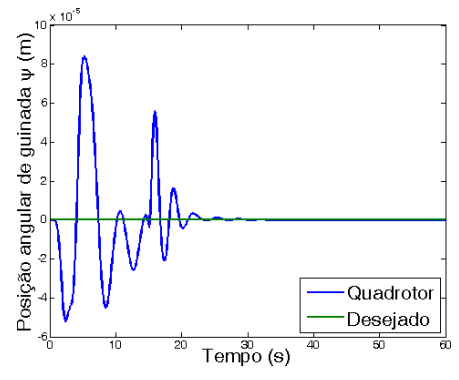

(c)

Figura 5: Posições angulares de rolagem 5(a), arfagem 5(b) e guinada 5(c).

processo de estabilização. Houveram também desvios nos valores dos ângulos de rolagem $(\phi)$, arfagem $(\theta)$ e guinada $(\psi)$. O desvio no ângulo $(\psi)$ é gerado para compensar a força de arrasto gerada pela aceleração do veículo.

\section{Conclusão}

No presente trabalho foi proposto o controle do quadrotor LARVANTAR, construído com auxílio de uma impressora 3D, utilizando um controlador PD. Os parâmetros modelo matemático da dinâmica do quadrotor foram identificados com auxílio de software CAD. O modelo foi avaliado por meio de simulação de voo, implementado utilizando o MATLAB ${ }^{\circledR}$. A simulação mostrou que o modelo matemático apresentado foi capaz de modelar de forma realista a posição e atitude do quadrotor. Os resultados das simulações mostraram também que o controlador PD foi eficiente na estabilização do quadrotor à altitude e atitude desejada. Como trabalho futuro, iremos implementar o sistema de controle de estabilidade no protótipo construído e comparar com resultados obtidos pela simulação.

\section{Agradecimentos}

Os autores gostariam de agradecer a FAPESP (Fundação de Amparo à Pesquisa do Estado de São Paulo), ao CNPq (Conselho Nacional de Desenvolvimento Científico e Tecnológico) e a CAPES (Coordenação de Aperfeiçoamento de Pessoal de Nível Superior) pelos auxílios financeiros propiciados.

\section{Referências}

[1] C. Bills, J. Chen and A. Saxena, Autonomous MAV flight in indoor environments using single image perspective cues, IEEE International Conference on Robotics and Automation, Shangai, China, (2011). 
[2] S. Bouabdallah, A. Noth and R. Siegwart, PID vs LQ control techniques applied to an indoor micro quadrotor, IEEE/RSJ International Conference on Intelligent Robots and Systems, Sendal, Japan, (2004).

[3] J. J. Craig, Introduction to Robotics: Mechanics and Control, Addison-Wesley Longman Publishing Co., Inc., Boston, MA, USA, Ed. 2, ISBN: 0201095289, (1989).

[4] E. Davis, B. E. Nizette and C. Yu, Development of a Low Cost Quadrotor Platform for Swarm Experiments, Chinese Control Conference, Xi'an,China, (2013).

[5] I. C. Dikmen, A. Arisoy and H. Temeltas, Attitude control of a quadcopter, International Conference on Recent Advances in Space Technologies, United States, (2010).

[6] J. Faigl, T. Krajnik, V. Vonasek, e L. Peuil, Surveillance Planning with Localization Uncertainty for UAVs, 3rd Israeli Conference on Robotics, (2010).

[7] I. C. S. Faria, W. M. Leão, M. H. Terra e R. S. Inoue, Identificação dos parâmetros do modelo dinâmico de um quadrotor, $67^{\circ}$ Reunião Anual da Sociedade Brasileira para o Progresso da Cicência, São Carlos, Brasil, (2015).

[8] K. Higuchi, T. Shimada and J. Rekimoto, Flying Sports Assistant: External Visual Imagery Representation for Sports Training, Proceedings of the $2^{\text {nd }}$ Augmented Human International Conference, (2011).

[9] G. Hunt, F. Mitzalis, T. Alhinai, P. A. Hooper and Mirko Kovac, 3D Printing with Flying Robots, IEEE International Conference on Robotics \& Automation, Hong Kong, China, (2014).

[10] T. Luukkonen, Modeling and control of quadcopter, Aalto University School of Science, (2011).

[11] R. A. F. Romero, E. Prestes, F. Osório e D. Wolf, Robótica Móvel, Editora LTC, Rio de Janeiro, (2014).

[12] J. L. Sanchez-Lopez, J. Pestana, P. de la Puente, R. Suarez-Fernandez and P. Campoy, A System for the Design and Development of Vision-based Multi-robot Quadrotor Swarms, Internatinal Conference on Unmanned Aircraft Systems, Orlando, USA, (2014).

[13] K. P. Valavanis and M. Kontitsis, A Historical Perspective on Unmanned Aerial Vehicles, Intelligent Systems, Control and Automation: Science and Engineering, Springer Netherlands, vol. 33, 15-46, DOI:10.1007/978-1-4020-6114-1, ISBN:978-14020-6113-4, (2007).

[14] Z. Zuo, Trajectory tracking control design with command-filtered compensation for a quadrotor, IET Control Theory Application, (2010). 